\title{
A Structural Property of Trees with an Application to Vertex-Arboricity
}

\author{
Ming-jia Wang and Jing-ti Han \\ Shanghai University of Finance and Economics, Shanghai 200433, China \\ Correspondence should be addressed to Ming-jia Wang; lxlsohu@126.com \\ Received 4 February 2017; Accepted 10 May 2017; Published 11 June 2017 \\ Academic Editor: Ruben Specogna
}

Copyright (C) 2017 Ming-jia Wang and Jing-ti Han. This is an open access article distributed under the Creative Commons Attribution License, which permits unrestricted use, distribution, and reproduction in any medium, provided the original work is properly cited.

\begin{abstract}
We provide a structural property of trees, which is applied to show that if a plane graph $G$ contains two edge-disjoint spanning trees, then its dual graph $G^{*}$ has the vertex-arboricity at most 2 . We also show that every maximal plane graph of order at least 4 contains two edge-disjoint spanning trees.
\end{abstract}

\section{Introduction}

All graphs considered in this paper are finite simple graphs. Given graph $G$, let $V(G), E(G),|G|$, and $\|G\|$ denote its vertex set, edge set, vertex number, and edge number, respectively. For a vertex $v \in V(G)$, let $d_{G}(v)$ denote the degree of $v$ in $G$. Moreover, let $\Delta(G)$ and $\delta(G)$ denote the maximum degree and minimum degree of $G$, respectively. A tree is a connected graph without cycles. A plane graph is a particular drawing of a planar graph on the Euclidean plane.

A subgraph $H$ of $G$ is called a spanning one if $V(H)=$ $V(G)$. A plane graph $G$ is called maximal if every face of $G$ is a triangle. A connected Eulerian graph is a connected one that contains no vertices of degree odd. The dual, denoted by $G^{*}$, of a plane graph $G$ is a plane graph whose vertices correspond to the faces of $G$ and edges correspond to the edges of $G$ in this way: if $e$ is an edge of $G$ incident to faces $f_{1}, f_{2}$, then the endpoints of the dual edge $e^{*} \in E\left(G^{*}\right)$ are vertices $v_{1}, v_{2}$ of $G^{*}$ that represent the faces of $f_{1}, f_{2}$ of $G$. The vertex-arboricity $a(G)$ of a graph $G$ is the minimum number of subsets into which $V(G)$ can be partitioned so that each subset induces a forest.

The vertex-arboricity of a graph was first introduced by Chartrand et al. [1], named as point-arboricity. Among other things, they proved that the vertex-arboricity of planar graphs is at most 3. Chartrand and Kronk [2] showed that this bound is sharp by presenting a planar graph of the vertex-arboricity 3. More generally, Kronk [3] showed that if $S$ is a surface with Euler genus $g$, then $a(S)=3$ and if $S$ is the sphere or the Klein bottle, then $a(S)=\lfloor(9+\sqrt{1+24 g}) / 4\rfloor$. Hara et al. [4] extended partially Kronk's result by proving that $l a(S)=3$ if $S$ is the projective plane or the torus, and $l a(S) \leq 4$ if $S$ is the Klein bottle. Here the vertex-arboricity of surface $S$ is defined to be the maximum of the vertex-arboricity of all graphs embeddable into $S$. Other results about the vertexarboricity of embedded graphs in the surface are referred to in [5-8].

The following theorem, due to Stein [9], characterizes completely maximal plane graphs with vertex-arboricity 2 .

Theorem 1. Let $G$ be a maximal plane graph with $|G| \geq 4$. Then $a(G)=2$ if and only if $G^{*}$ is Hamiltonian.

Hakimi and Schmeichel [10] extended Stein's theorem to the following form.

Theorem 2. Let $G$ be a plane graph. Then $a(G)=2$ if and only if $G^{*}$ contains a connected Eulerian spanning subgraph.

Note that determining whether a graph to have a connected Eulerian spanning subgraph is quite difficult. Thus a natural question is as follows: which plane graphs have 
a connected Eulerian spanning subgraph? In this paper, we provide a sufficient condition about the problem.

\section{Structural Property}

Let $P=(x, y)$ denote a path from vertex $x$ to vertex $y$. We call a vertex of degree 1 a leaf in a tree. The following lemma is of interest by itself.

Theorem 3. Let $T$ be a tree with $|T| \geq 2$ and $n \geq 1$ be an integer. If $S \subseteq V(T)$ with $|S|=2 n \leq|T|$, then $S$ can be partitioned into two sets $X=\left\{x_{1}, x_{2}, \ldots, x_{n}\right\}$ and $Y=$ $\left\{y_{1}, y_{2}, \ldots, y_{n}\right\}$ such that $T$ contains $n$ edge-disjoint paths $P_{i}=$ $\left(x_{i}, y_{i}\right), i=1,2, \ldots, n$.

Proof. The proof is proceeded by induction on the order of $T$. If $2 \leq|T| \leq 3$, then $T$ is a path of length 1 or 2 . Note that $n=1$ in this case, so the theorem holds trivially. Suppose that $T$ is a tree with $|T| \geq 4$. Let $S$ be a subset of $V(T)$ with $|S|=2 n \leq|T|$. If $T$ contains a leaf $v$ which is not in $S$, then $|S| \leq|T|-1$. Let $T^{\prime}=T-v$. Then $T^{\prime}$ is a tree of order $|T|-1$ and $S \subseteq V\left(T^{\prime}\right)$ satisfies $|S|=2 n \leq|T|-1=\left|T^{\prime}\right|$. By the induction hypothesis, $S$ can be partitioned into two sets $X=$ $\left\{x_{1}, x_{2}, \ldots, x_{n}\right\}$ and $Y=\left\{y_{1}, y_{2}, \ldots, y_{n}\right\}$ such that $T^{\prime}$ contains $n$ edge-disjoint paths $P_{i}=\left(x_{i}, y_{i}\right), i=1,2, \ldots, n$. Clearly, $S=X \cup Y$ also is a required partition of $T$.

So assume that $S$ contains all the leaves of $T$. If there exist two leaves $x$ and $y$ adjacent to a common vertex $u$, we let $T^{\prime}=$ $T-\{x, y\}$ and $S^{\prime}=S \backslash\{x, y\}$. Then $T^{\prime}$ is a tree of order $|T|-2$ and $S^{\prime} \subseteq V\left(T^{\prime}\right)$ satisfies $\left|S^{\prime}\right|=|S|-2=2 n-2 \leq|T|-2=\left|T^{\prime}\right|$. By the induction hypothesis, $S^{\prime}$ can be partitioned into two sets $X^{\prime}=\left\{x_{1}, x_{2}, \ldots, x_{n-1}\right\}$ and $Y^{\prime}=\left\{y_{1}, y_{2}, \ldots, y_{n-1}\right\}$ such that $T^{\prime}$ contains $n-1$ edge-disjoint paths $P_{i}^{\prime}=\left(x_{i}, y_{i}\right), i=$ $1,2, \ldots, n-1$. Defining $P_{n}=x u y, P_{i}=P_{i}^{\prime}$ for $i=1,2, \ldots, n-1$, $X=X^{\prime} \cup\{x\}$, and $Y=Y^{\prime} \cup\{y\}$, we obtain a desired partition $S=X \cup Y$ of $T$.

Now suppose that every vertex of $T$ is adjacent to at most one leaf. Let $Q=v_{1} v_{2} v_{3} \cdots v_{k}$ be a longest path in $T$. Then it is easy to see that $k \geq 4$ and $v_{1}, v_{k}$ are leaves. If $v_{2}$ is adjacent to some vertex, $u_{1}$, different from $v_{1}$ and $v_{3}$, then $u_{1}$ is not a leaf by the assumption. There exists a vertex $u_{2}$, other than $v_{2}$, adjacent to $u_{1}$. However, $Q^{\prime}=u_{2} u_{1} v_{2} v_{3} \cdots v_{k}$ is a path whose length is greater than that of $Q$, contradicting the choice of $Q$. Hence it follows that $d_{T}\left(v_{2}\right)=2$. Similarly, we derive that $d_{T}\left(v_{k-1}\right)=2$.

If $v_{2} \in S$, we define $T^{\prime}=T-\left\{v_{1}, v_{2}\right\}$ and $S^{\prime}=S \backslash\left\{v_{1}, v_{2}\right\}$. Then $S^{\prime} \subseteq V\left(T^{\prime}\right)$ satisfies $\left|S^{\prime}\right|=|S|-2=2 n-2 \leq|T|-2=\left|T^{\prime}\right|$. By the induction hypothesis, $S^{\prime}$ admits a required partition $X^{\prime} \cup Y^{\prime}$ with $\left|X^{\prime}\right|=\left|Y^{\prime}\right|=n-1$ so that $T^{\prime}$ contains $n-1$ edge-disjoint paths $P_{1}, P_{2}, \ldots, P_{n-1}$. In $T$, we define $P_{n}=v_{1} v_{2}$, $X=X^{\prime} \cup\left\{v_{1}\right\}$, and $Y=Y^{\prime} \cup\left\{v_{2}\right\}$.

If $v_{2} \notin S$, then $|S| \leq|T|-1$. Let $T^{\prime}=T-v_{2}+v_{1} v_{3}$ and $S^{\prime}=S$. Then $T^{\prime}$ is a tree of order $|T|-1$ and $S^{\prime} \subseteq V\left(T^{\prime}\right)$ with $\left|S^{\prime}\right|=2 n \leq|T|-1=\left|T^{\prime}\right|$. By the induction hypothesis, $S^{\prime}$ can be partitioned into two sets $X^{\prime}=\left\{x_{1}, x_{2}, \ldots, x_{n}\right\}$ and $Y^{\prime}=$ $\left\{y_{1}, y_{2}, \ldots, y_{n}\right\}$ such that $T^{\prime}$ contains $n$ edge-disjoint paths $P_{i}^{\prime}=\left(x_{i}, y_{i}\right), i=1,2, \ldots, n$. Since $v_{1}$ is a leaf of $T^{\prime}, v_{1}$ is an end of some path $P_{i^{0}}^{\prime}$. Furthermore, $v_{1} v_{3} \in E\left(P_{i^{0}}^{\prime}\right)$. Without loss of generality, suppose that $i^{0}=n$ and $P_{n}=v_{1} v_{3} z_{1} z_{2} \cdots z_{m}$. In $T$, we let $X=X^{\prime}, Y=Y^{\prime}, P_{n}=v_{1} v_{2} v_{3} z_{1} z_{2} \cdots z_{m}$, and $P_{i}=P_{i}^{\prime}$ for $i=1,2, \ldots, n-1$. A desired partition of $S$ in $T$ is established. This proves the theorem.

By means of Theorem 3, we can give a simple proof for the following result.

Theorem 4. Let $G$ be a graph with $|G| \geq 4$. If $G$ contains two edge-disjoint spanning trees, then $G$ contains a connected Eulerian spanning subgraph.

Proof. Suppose that $T_{1}$ and $T_{2}$ are two edge-disjoint spanning trees of $G$. Then $V\left(T_{1}\right)=V\left(T_{2}\right)=V(G)$. Let $S^{*}$ denote the subset of vertices of degree odd in $T_{1}$. Then $\left|S^{*}\right|$ is even. Since $T_{1}$ contains at least two leaves, $2 \leq\left|S^{*}\right|=2 n \leq\left|T_{1}\right|=\left|T_{2}\right|$. Since $S^{*} \subseteq V\left(T_{2}\right)$, by Theorem $3, S^{*}$ can be partitioned into two sets $X=\left\{x_{1}, x_{2}, \ldots, x_{n}\right\}$ and $Y=\left\{y_{1}, y_{2}, \ldots, y_{n}\right\}$ such that $T_{2}$ contains $n$ edge-disjoint paths $P_{i}=\left(x_{i}, y_{i}\right)$, $i=1,2, \ldots, n$.

Let $M$ denote the subgraph of $G$ induced by the edge set $E\left(P_{1}\right) \cup \cdots \cup E\left(P_{n}\right)$. Let $H=T_{1} \cup M . T_{1}$ is connected and spanning, so is $H$. Let $v \in V(H)=V(G)$. It is easy to observe that $d_{H}(v)=d_{T_{1}}(v)+d_{M}(v)$. If $v \notin S^{*}$, then both $d_{T_{1}}(v)$ and $d_{M}(v)$ are even, and hence $d_{H}(v)$ is even. If $v \in S^{*}$, then both $d_{T_{1}}(v)$ and $d_{M}(v)$ are odd, and so $d_{H}(v)$ is even. It follows that $H$ is Eulerian. Thus $H$ is a connected Eulerian spanning subgraph of $G$. The proof of the theorem is complete.

Combining Theorems 2 and 4, we obtain the main result in this paper.

Theorem 5. Let $G$ be a plane graph. If $G^{*}$ contains two edgedisjoint spanning trees, then $a(G)=2$.

\section{Spanning Trees in Maximal Plane Graphs}

Let $G$ be a maximal plane graph. Then the following properties (a)-(d) hold:

(a) $\|G\|=3|G|-6$;

(b) $3 \leq \delta(G) \leq 5$ if $|G| \geq 4$;

(c) Each of the faces in $G$ is of degree 3;

(d) If $|G|=3$, then $G$ is isomorphic to $K_{3}$; if $|G|=4$, then $G$ is isomorphic to $K_{4}$; if $|G|=5$, then $G$ is isomorphic to $K_{5}-e$, where $e$ is any edge of $K_{5}$. In particular, when $|G| \geq 5$, we have that $\Delta(G) \geq 4$.

Theorem 6. Every maximal plane graph $G$ with $\Delta(G) \geq 3$ contains two edge-disjoint spanning trees.

Proof. We prove the theorem by induction on the vertex number $|G|$. Since $\Delta(G) \geq 3$, it follows that $|G| \geq 4$. If $|G|=4$, then $G$ is isomorphic to $K_{4}$, and $K_{4}$ can be easily edgepartitioned into two edge-disjoint spanning trees. Hence the basis step of induction is established. Let $G$ be a maximal plane graph with $|G| \geq 5$. So $\Delta(G) \geq 4$. By the above property (b), $G$ contains a vertex $v$ with $3 \leq d_{G}(v) \leq 5$. Set $k=d_{G}(v)$. Let $v_{0}, v_{1}, \ldots, v_{k-1}$ denote the neighbors of $v$ in $G$ in clockwise 
order and $f_{0}, f_{1}, \ldots, f_{k-1}$ denote the incident faces of $v$ in $G$ in clockwise order with $v v_{i}, v v_{i+1}$ as two boundary edges of $f_{i}$ for $i=0,1, \ldots, k-1$. Here all the indices are taken modulo $k$. Note that $f_{0}, f_{1}, \ldots, f_{k-1}$ are all 3 faces.

The proof is split into the following three cases, depending on the size of $k$.

Case $1(k=3)$. Let $G^{\prime}=G-v$. Then $G^{\prime}$ is a maximal plane graph with $\left|G^{\prime}\right|<|G|$ and $\Delta\left(G^{\prime}\right) \geq \Delta(G)-1 \geq 3$. By the induction hypothesis, $G^{\prime}$ contains two edge-disjoint spanning trees $T_{1}^{\prime}$ and $T_{2}^{\prime}$. Let $T_{1}=T_{1}^{\prime}+v v_{1}$ and $T_{2}=T_{2}^{\prime}+v v_{2}$. Obviously, $T_{i}$ is a spanning tree of $G$ for $i=1,2$, and $E\left(T_{1}\right) \cap E\left(T_{2}\right)=\emptyset$. Thus, the theorem holds in this situation.

Case 2 $(k=4)$. In view of the planarity of $G$, at least one of $v_{0} v_{2}$ and $v_{1} v_{3}$ does not belong to $E(G)$. Without loss of generality, assume that $v_{0} v_{2} \notin E(G)$. Let $G^{\prime}=G-v+v_{0} v_{2}$. Then $G^{\prime}$ is a maximal plane graph with $\left|G^{\prime}\right|<|G|$ and $\Delta\left(G^{\prime}\right) \geq \Delta(G)-1 \geq$ 3. By the induction hypothesis, $G^{\prime}$ contains two edge-disjoint spanning trees $T_{1}^{\prime}$ and $T_{2}^{\prime}$. If $v_{0} v_{2} \notin E\left(T_{1}^{\prime}\right) \cup E\left(T_{2}^{\prime}\right)$, then we define $T_{i}=T_{i}^{\prime}+v v_{i}$ for $i=1,2$. If $v_{0} v_{2} \in E\left(T_{1}^{\prime}\right)$, then we define $T_{1}=\left(T_{1}^{\prime}-v_{0} v_{2}\right)+\left\{v v_{0}, v v_{2}\right\}$ and $T_{2}=T_{2}^{\prime}+v v_{1}$. If $v_{0} v_{2} \in E\left(T_{2}^{\prime}\right)$, then we define $T_{1}=T_{1}^{\prime}+v v_{1}$ and $T_{2}=\left(T_{2}^{\prime}-\right.$ $\left.v_{0} v_{2}\right)+\left\{v v_{0}, v v_{2}\right\}$. It is easy to inspect that $T_{1}$ and $T_{2}$ are two edge-disjoint spanning trees of $G$ in each of the above three cases.

Case $3(k=5)$. Again, by the planarity of $G$, there exists a vertex $v_{i}$, say $i=0$, such that $v_{0} v_{2}, v_{0} v_{3} \notin E(G)$. Let $G^{\prime}=G-$ $v+\left\{v_{0} v_{2}, v_{0} v_{3}\right\}$. Then $G^{\prime}$ is a maximal plane graph with $\left|G^{\prime}\right|<$ $|G|$ and $\Delta\left(G^{\prime}\right) \geq \Delta(G)-1 \geq 3$. By the induction hypothesis, $G^{\prime}$ contains two edge-disjoint spanning trees $T_{1}^{\prime}$ and $T_{2}^{\prime}$. We have to consider the following subcases.

Case 3.1. At least one of $v_{0} v_{2}$ and $v_{0} v_{3}$ does not belong to $E\left(T_{1}^{\prime}\right) \cup E\left(T_{2}^{\prime}\right)$, say $v_{0} v_{3} \notin E\left(T_{1}^{\prime}\right) \cup E\left(T_{2}^{\prime}\right)$.

If $v_{0} v_{2} \notin E\left(T_{1}^{\prime}\right) \cup E\left(T_{2}^{\prime}\right)$, then we set $T_{i}=T_{i}^{\prime}+v v_{i}$ for $i=1,2$. If $v_{0} v_{2} \in E\left(T_{1}^{\prime}\right)$, then we set $T_{1}=\left(T_{1}^{\prime}-v_{0} v_{2}\right)+\left\{v v_{0}, v v_{2}\right\}$ and $T_{2}=T_{2}^{\prime}+v v_{3}$. If $v_{0} v_{2} \in E\left(T_{2}^{\prime}\right)$, then we set $T_{1}=T_{1}^{\prime}+v v_{1}$ and $T_{2}=\left(T_{2}^{\prime}-v_{0} v_{2}\right)+\left\{v v_{0}, v v_{2}\right\}$.

Case $3.2\left(v_{0} v_{2}, v_{0} v_{3} \in E\left(T_{1}^{\prime}\right) \cup E\left(T_{2}^{\prime}\right)\right)$. We need to deal with the following two subcases by symmetry.

Case 3.2.1 $\left(v_{0} v_{2}, v_{0} v_{3} \in E\left(T_{i}^{\prime}\right)\right.$ for some $\left.i \in\{1,2\}\right)$. Without loss of generality, assume that $i=1$. It is enough to set $T_{1}=$ $\left(T_{1}^{\prime}-\left\{v_{0} v_{2}, v_{0} v_{3}\right\}\right)+\left\{v v_{0}, v v_{2}, v v_{3}\right\}$ and $T_{2}=T_{2}^{\prime}+v v_{1}$.

Case 3.2.2 $\left(v_{0} v_{2} \in E\left(T_{1}^{\prime}\right)\right.$ and $\left.v_{0} v_{3} \in E\left(T_{2}^{\prime}\right)\right)$. Let $A_{1}$ and $B_{1}$ denote the two components of $T_{1}^{\prime}-v_{0} v_{2}$ with $v_{0} \in V\left(A_{1}\right)$ and $v_{2} \in V\left(B_{1}\right)$, and $A_{2}$ and $B_{2}$ denote the two components of $T_{2}^{\prime}-v_{0} v_{3}$ with $v_{0} \in V\left(A_{2}\right)$ and $v_{3} \in V\left(B_{2}\right)$. Since $v_{0} v_{2}$ is a cut edge of $T_{1}^{\prime}$, it follows that $v_{1}$ belongs to exactly one of $V\left(A_{1}\right)$ and $V\left(B_{1}\right)$. Similarly, $v_{4}$ belongs to exactly one of $V\left(A_{2}\right)$ and $V\left(B_{2}\right)$.

If $v_{1} \in V\left(A_{1}\right)$, then we define $T_{1}=\left(T_{1}^{\prime}-v_{0} v_{2}\right)+\left\{v v_{1}, v v_{2}\right\}$ and $T_{2}=\left(T_{2}^{\prime}-v_{0} v_{3}\right)+\left\{v v_{0}, v v_{3}\right\}$.

If $v_{4} \in V\left(A_{2}\right)$, then we define $T_{1}=\left(T_{1}^{\prime}-v_{0} v_{2}\right)+\left\{v v_{0}, v v_{2}\right\}$ and $T_{2}=\left(T_{2}^{\prime}-v_{0} v_{3}\right)+\left\{v v_{3}, v v_{4}\right\}$.
Now assume that $v_{1} \in V\left(B_{1}\right)$ and $v_{4} \in V\left(B_{2}\right)$. This implies that $v_{0} v_{1} \notin E\left(T_{1}^{\prime}\right)$ and $v_{0} v_{4} \notin E\left(T_{2}^{\prime}\right)$. If $v_{0} v_{1} \notin E\left(T_{2}^{\prime}\right)$, then we define $T_{1}=\left(T_{1}^{\prime}-v_{0} v_{2}\right)+\left\{v_{0} v_{1}, v v_{1}\right\}$ and $T_{2}=\left(T_{2}^{\prime}-v_{0} v_{3}\right)+$ $\left\{v v_{0}, v v_{3}\right\}$. If $v_{0} v_{4} \notin E\left(T_{1}^{\prime}\right)$, then we define $T_{1}=\left(T_{1}^{\prime}-v_{0} v_{2}\right)+$ $\left\{v v_{0}, v v_{2}\right\}$ and $T_{2}=\left(T_{2}^{\prime}-v_{0} v_{3}\right)+\left\{v_{0} v_{4}, v v_{4}\right\}$. Otherwise, $v_{0} v_{1} \in$ $E\left(T_{2}^{\prime}\right)$ and $v_{0} v_{4} \in E\left(T_{1}^{\prime}\right)$. It suffices to define $T_{1}=\left(T_{1}^{\prime}-v_{0} v_{2}\right)+$ $\left\{v v_{2}, v v_{4}\right\}$ and $T_{2}=\left(T_{2}^{\prime}-v_{0} v_{3}\right)+\left\{v v_{1}, v v_{3}\right\}$.

It is easy to inspect that $T_{1}$ and $T_{2}$ are two edge-disjoint spanning trees of $G$ in every possible case above. This completes the proof of the theorem.

The following consequence follows immediately from Theorems 4 and 6.

Corollary 7. Every maximal planar graph $G$ with $|G| \geq 4$ contains a connected Eulerian spanning subgraph.

\section{Conflicts of Interest}

The authors declare that they have no conflicts of interest.

\section{References}

[1] G. Chartrand, H. V. Kronk, and C. E. Wall, "The pointarboricity of a graph," Israel Journal of Mathematics, vol. 6, pp. 169-175, 1968.

[2] G. Chartrand and H. V. Kronk, "The point-arboricity of planar graphs," Journal of the London Mathematical Society. Second Series, vol. 44, pp. 612-616, 1969.

[3] H. V. Kronk, "An analogue to the Heawood map-colouring problem," Journal of the London Mathematical Society. Second Series, vol. 1, pp. 750-752, 1969.

[4] M. Hara, Y. Ohyama, and S. Yamashita, "On the linear vertexarboricity of a surface," Journal of Combinatorial Mathematics and Combinatorial Computing, vol. 18, pp. 3-10, 1995.

[5] M. Chen, L. Huang, and W. Wang, "List vertex-arboricity of toroidal graphs without 4-cycles adjacent to 3-cycles," Discrete Mathematics, vol. 339, no. 10, pp. 2526-2535, 2016.

[6] M. Chen, A. Raspaud, and W. Wang, "Vertex-arboricity of planar graphs without intersecting triangles," European Journal of Combinatorics, vol. 33, no. 5, pp. 905-923, 2012.

[7] D. Huang and W. Wang, "Vertex arboricity of planar graphs without chordal 6-cycles," International Journal of Computer Mathematics, vol. 90, no. 2, pp. 258-272, 2013.

[8] A. Raspaud and W. Wang, "On the vertex-arboricity of planar graphs," European Journal of Combinatorics, vol. 29, no. 4, pp. 1064-1075, 2008.

[9] S. K. Stein, "B-set and planar maps", Pacific Journal of Mathematics, vol. 37, pp. 217-224, 1971.

[10] S. L. Hakimi and E. F. Schmeichel, "A note on the vertex arboricity of a graph," SIAM Journal on Discrete Mathematics, vol. 2, no. 1, pp. 64-67, 1989. 


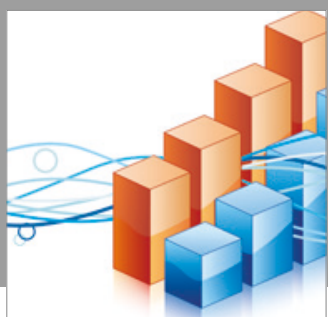

Advances in

Operations Research

vatersals

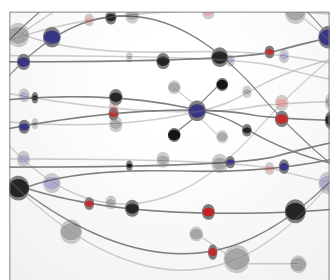

\section{The Scientific} World Journal
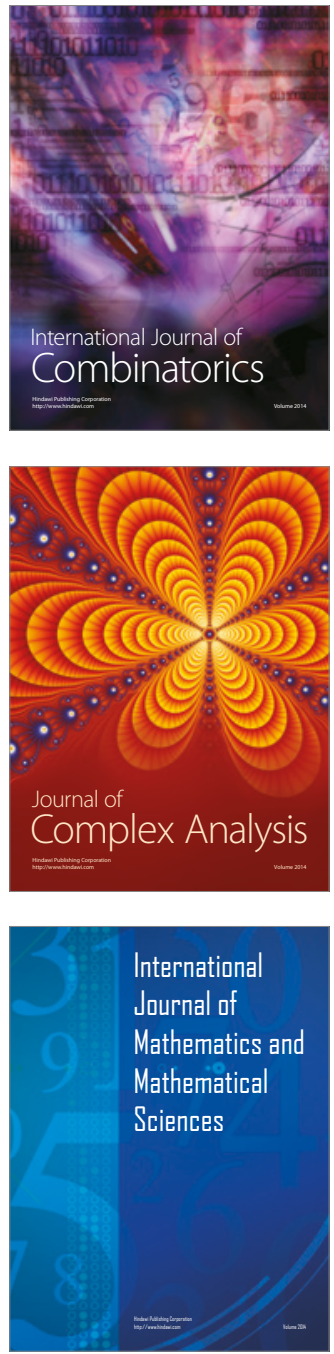
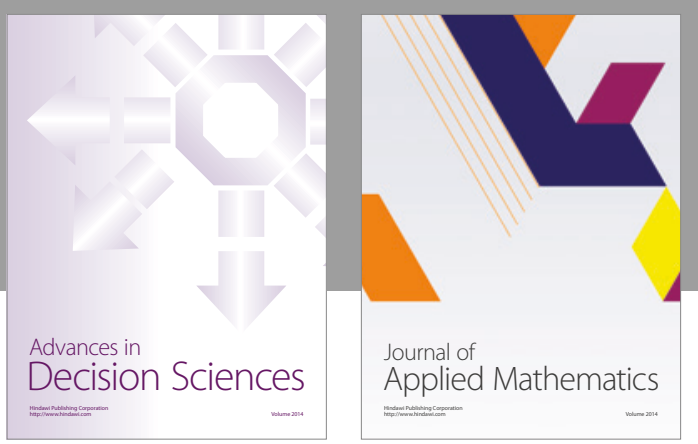

Algebra

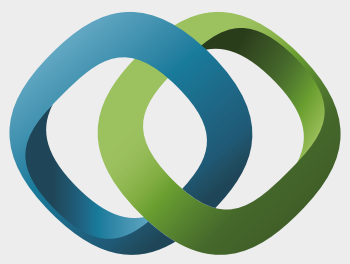

\section{Hindawi}

Submit your manuscripts at

https://www.hindawi.com
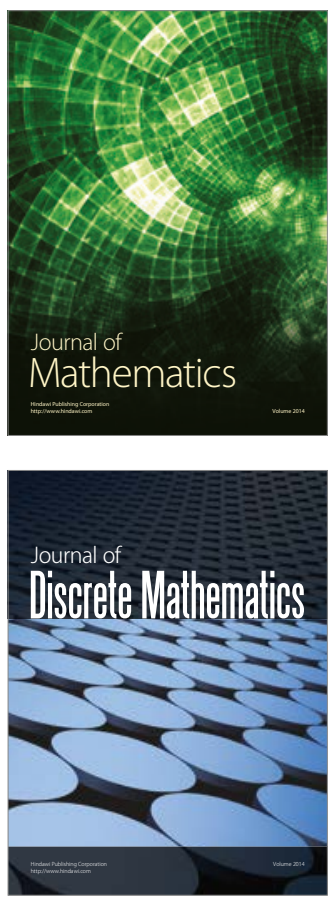

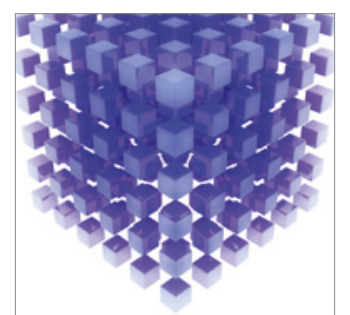

Mathematical Problems in Engineering
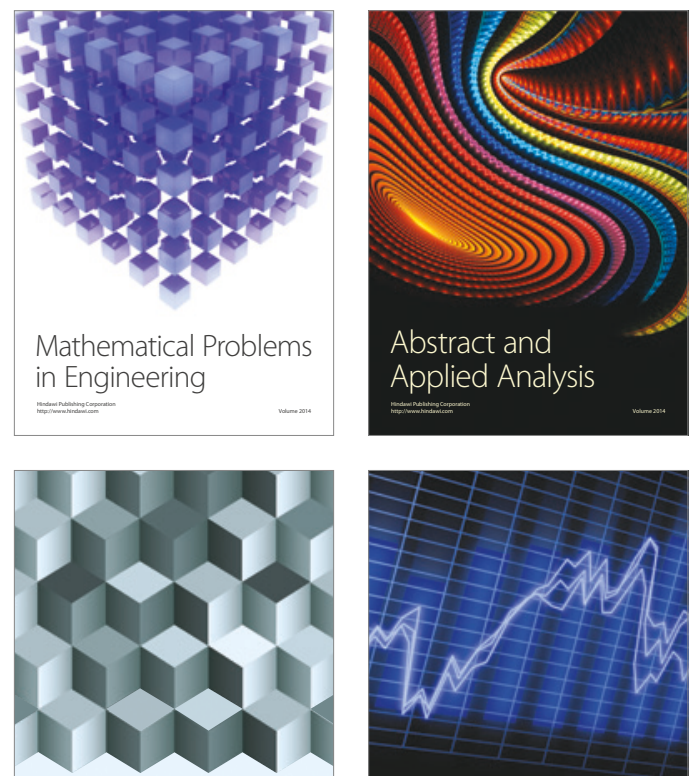

Journal of

Function Spaces

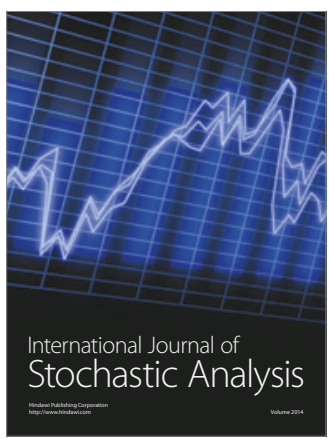

Probability and Statistics
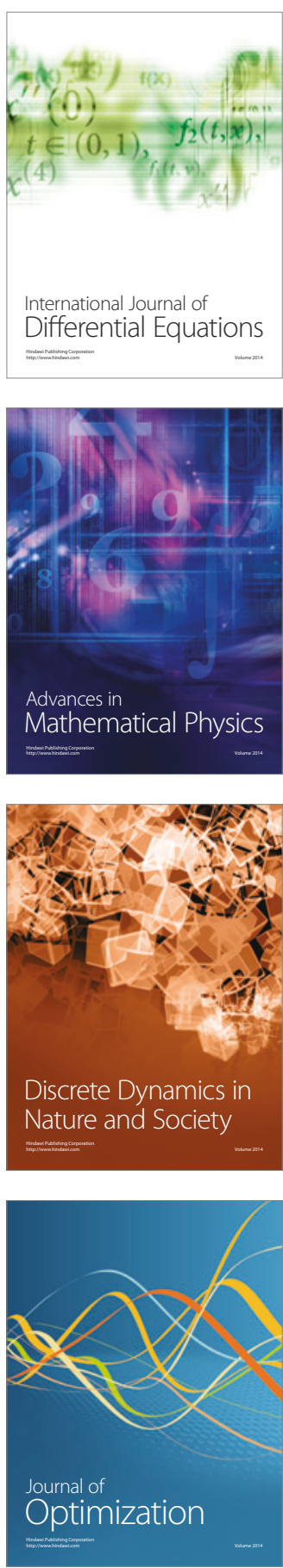